\title{
Probing the Triple Higgs Self-Interaction at the Large Hadron Collider
}

\author{
Jeong Han Kim, ${ }^{1}$ Kyoungchul Kong, ${ }^{1}$ Konstantin T. Matchev, ${ }^{2}$ and Myeonghun Park ${ }^{3}$ \\ ${ }^{1}$ Department of Physics and Astronomy, University of Kansas, Lawrence, Kansas 66045, USA \\ ${ }^{2}$ Physics Department, University of Florida, Gainesville, Florida 32611, USA \\ ${ }^{3}$ Institute of Convergence Fundamental Studies and School of Liberal Arts, Seoultech, Seoul 01811, Korea
}

(Received 21 August 2018; published 6 March 2019)

\begin{abstract}
We propose a novel kinematic method to expedite the discovery of the double Higgs $(h h)$ production in the $\ell^{+} \ell^{-} b \bar{b}+E_{T}$ final state. We make full use of recently developed kinematic variables, as well as the variables "Topness" for the dominant background (top quark pair production) and "Higgsness" for the signal. We obtain a significant increase in sensitivity compared to the previous analyses that used sophisticated algorithms like boosted decision trees or neutral networks. The method can be easily generalized to resonant $h h$ production as well as other nonresonant channels.
\end{abstract}

DOI: 10.1103/PhysRevLett.122.091801

Introduction.-The discovery of the Higgs boson $(h)$ with a mass $m_{h}=125 \mathrm{GeV}[1,2]$ jumpstarted a comprehensive program of the precision measurements of all Higgs couplings. The current results for the couplings to fermions and gauge bosons [3] appear to be in agreement with the standard model (SM) predictions. However, probing the triple and quartic Higgs self-couplings is notoriously difficult [4-11]. Yet, the knowledge of those couplings is crucial for understanding the exact mechanism of electroweak symmetry breaking and the origin of mass in our universe.

The Higgs self-interaction is parametrized as follows:

$$
V=\frac{m_{h}^{2}}{2} h^{2}+\kappa_{3} \lambda_{3}^{\mathrm{SM}} v h^{3}+\frac{1}{4} \kappa_{4} \lambda_{4}^{\mathrm{SM}} h^{4},
$$

where $\lambda_{3}^{\mathrm{SM}}=\lambda_{4}^{\mathrm{SM}}=\left[m_{h}^{2} /\left(2 v^{2}\right)\right]$ are the SM values, $\kappa_{3}$ and $\kappa_{4}$ parametrize deviations from those, and $v \approx 256 \mathrm{GeV}$ is the Higgs vacuum expectation value. In order to access $\kappa_{3}$ $\left(\kappa_{4}\right)$, one has to measure the process of double (triple) Higgs boson production at the Large Hadron Collider (LHC) or future colliders, possibly with high luminosity (HL). Due to the small signal cross section $\left(\sigma_{h h}\right)$, it is necessary to combine as many different channels as possible. One specific process, $h h \rightarrow(b \bar{b})\left(W^{ \pm} W^{\mp}\right)$, has so far been relatively overlooked, due to the large SM background cross section $\sigma_{\text {bknd }} \sim 10^{5} \sigma_{h h}$, which is predominantly due to top quark pair production $(t \bar{t})$. In particular, there have been very few studies on the resulting dilepton final state [8-12]. The existing analyses employ sophisticated algorithms \{neutral network (NN) [9], deep

Published by the American Physical Society under the terms of the Creative Commons Attribution 4.0 International license. Further distribution of this work must maintain attribution to the author(s) and the published article's title, journal citation, and DOI. Funded by SCOAP . neutral network (DNN) [10], boosted decision tree (BDT) $[11,12]$, etc. $\}$ to increase the signal sensitivity, but show somewhat pessimistic results, with a significance no better than $1 \sigma$ at the HL-LHC with $3 \mathrm{ab}^{-1}$ luminosity [9-12].

In this Letter, we propose a novel method to enhance the signal significance for $h h$ production in the dilepton channel. The idea is to maximize the use of kinematic information for the dominant background (dilepton $t \bar{t}$ production). For this purpose, we utilize the class of kinematic variables, which were specifically designed for the dilepton $t \bar{t}$ topology [13-17]. In addition, we introduce a discriminator against signal, which we refer to as "Topness," following Ref. [18], and an analogous discriminator against background, called "Higgsness." We do not use matrix elements or any of the above mentioned complicated algorithms. The method leads to a surprisingly high significance compared to existing results. We will first carry out an analysis for the case of a SM-like Higgs boson, $\kappa_{3}=1$, before extending to non-SM values.

Method.-Our method relies on two new kinematics functions, Topness and Higgsness, which respectively characterize features of $t \bar{t}$ and $h h$ events, and two less commonly used variables, subsystem $M_{T 2}$ (or $M_{2}$ ) [13,16,19] for $t \bar{t}$ and subsystem $\sqrt{\hat{s}}_{\text {min }}\left(\right.$ or $M_{1}$ ) [14-16] for $h h$ production. Topness provides a degree of consistency for a given event to dilepton $t \bar{t}$ production, in which there are six unknowns (the three momenta of the two neutrinos, $\vec{p}_{\nu}$ and $\vec{p}_{\bar{\nu}}$ ) and four on-shell constraints, $m_{t}, m_{\bar{t}}$, $m_{W^{+}}$, and $m_{W^{-}}$. The neutrino momenta can be fixed by minimizing the following quantity

$$
\begin{aligned}
\chi_{i j}^{2} \equiv \min _{\vec{p}_{T}=\vec{p}_{\nu T}+\vec{p}_{\bar{\nu} T}}\left(\frac{\left(m_{b_{i} \ell^{+} \nu}^{2}-m_{t}^{2}\right)^{2}}{\sigma_{t}^{4}}+\frac{\left(m_{\ell^{+} \nu}^{2}-m_{W}^{2}\right)^{2}}{\sigma_{W}^{4}}\right. \\
\left.+\frac{\left(m_{b_{j} \ell^{-} \bar{\nu}}^{2}-m_{t}^{2}\right)^{2}}{\sigma_{t}^{4}}+\frac{\left(m_{\ell^{-}-\bar{\nu}}^{2}-m_{W}^{2}\right)^{2}}{\sigma_{W}^{4}}\right),
\end{aligned}
$$


subject to the missing transverse momentum constraint, $\vec{p}_{T}=\vec{p}_{\nu T}+\vec{p}_{\bar{\nu} T}$. We use MINUIT for the minimization in our analysis [20]. Since there is a twofold ambiguity in the paring of a $b$ quark and a lepton, we define "Topness" as the smaller of two $\chi^{2} \mathrm{~s}$,

$$
T \equiv \min \left(\chi_{12}^{2}, \chi_{21}^{2}\right)
$$

In double Higgs production, the two $b$ quarks arise from a Higgs decay $(h \rightarrow b \bar{b})$, and therefore their invariant mass $m_{b b}$ can be used as a first cut to enhance the signal sensitivity. For the decay of the other Higgs boson, $h \rightarrow W^{ \pm} W^{\mp}$, we define "Higgsness" as follows:

$$
\begin{aligned}
H \equiv & \min \left[\frac{\left(m_{\ell^{+} \ell^{-} \nu \bar{\nu}}^{2}-m_{h}^{2}\right)^{2}}{\sigma_{h_{\ell}}^{4}}+\frac{\left(m_{\nu \bar{\nu}}^{2}-m_{\nu \bar{\nu} \text {,peak }}^{2}\right)^{2}}{\sigma_{\nu}^{4}}\right. \\
& +\min \left(\frac{\left(m_{\ell^{+} \nu}^{2}-m_{W}^{2}\right)^{2}}{\sigma_{W}^{4}}+\frac{\left(m_{\ell^{-} \bar{\nu}}^{2}-m_{W_{\text {,peak }}^{*}}^{2}\right)^{2}}{\sigma_{W^{*}}^{4}},\right. \\
& \left.\left.\frac{\left(m_{\ell^{-} \bar{\nu}}^{2}-m_{W}^{2}\right)^{2}}{\sigma_{W}^{4}}+\frac{\left(m_{\ell^{+} \nu}^{2}-m_{W_{\text {,peak }}^{*}}^{2}\right)^{2}}{\sigma_{W^{*}}^{4}}\right)\right],
\end{aligned}
$$

where $m_{W^{*}}$ is the invariant mass of the lepton-neutrino pair which resulted from the off-shell $W$. It satisfies $0 \leq m_{W^{*}} \leq$ $m_{h}-m_{W}$ and the peak of its distribution is at $m_{W^{*}}^{\text {peak }}=$ $(1 / \sqrt{3}) \sqrt{2\left(m_{h}^{2}+m_{W}^{2}\right)-\sqrt{m_{h}^{4}+14 m_{h}^{2} m_{W}^{2}+m_{W}^{4}}} \cdot m_{\nu \bar{\nu}}^{\text {peak }}=$ $m_{\ell \ell}^{\text {peak }} \approx 30 \mathrm{GeV}$ is the location of the peak in the $\left[d \sigma / d m_{\nu \bar{\nu}}\right]$ or $\left[d \sigma / d m_{\ell \ell}\right]$ distribution, which is bounded from above by $m_{\nu \bar{\nu}}^{\max }=m_{\ell \ell}^{\max }=\sqrt{m_{h}^{2}-m_{W}^{2}}$. The phase space distribution of $\left[d \sigma / d m_{\nu \bar{\nu}}\right]$ is given by $\left[(d \sigma) /\left(d m_{\nu \bar{\nu}}\right)\right] \propto \int d m_{W^{*}}^{2} \lambda^{1 / 2}\left(m_{h}^{2}, m_{W}^{2}, m_{W^{*}}^{2}\right) f\left(m_{\nu \bar{\nu}}\right)$, where $\lambda(x, y, z)=x^{2}+y^{2}+z^{2}-2 x y-2 y z-2 z x$ is the twobody phase space function and $f(m)$ is the invariant mass distribution of the antler topology [21-24] with $h \rightarrow W W^{*} \rightarrow \ell^{+} \ell^{-} \nu \bar{\nu}$

$$
f(m) \sim \begin{cases}\eta m, & 0 \leq m \leq e^{-\eta} E, \\ m \ln (E / m), & e^{-\eta} E \leq m \leq E,\end{cases}
$$

where now the endpoint $E$ and the parameter $\eta$ are defined in terms of the particle masses as $E=\sqrt{m_{W m_{W^{*}} e^{\eta}}}$ and $\cosh \eta=\left[\left(m_{h}^{2}-m_{W}^{2}-m_{W^{*}}^{2}\right) /\left(2 m_{W} m_{W^{*}}\right)\right]$. The actual peak of $30 \mathrm{GeV}$ is slightly less than the result for pure phase space due to a helicity suppression in the $W-\ell-\nu$ vertex.

The $\sigma$ values in Eq. (2) and Eq. (4) are associated with experimental uncertainties and intrinsic particle widths, but in principle, they can be treated as free parameters and one can tune them using NN, BDT, etc. In our numerical study, we use $\sigma_{t}=5 \mathrm{GeV}, \sigma_{W}=5 \mathrm{GeV}, \sigma_{W^{*}}=5 \mathrm{GeV}$, $\sigma_{h_{\ell}}=2 \mathrm{GeV}$, and $\sigma_{\nu}=10 \mathrm{GeV}$. The main contribution in Eq. (4) comes from the on-shell conditions for the Higgs and the $W$, while the effects of the invariant mass of the two neutrinos and the off-shell $W$ are minor.

Along with Higgsness and Topness, we adopt the subsystem $\hat{s}_{\min }^{(\ell \ell)}$ for $h \rightarrow W^{ \pm} W^{* \mp} \rightarrow \ell^{+} \ell^{-} \nu \bar{\nu}[14,15]$ and the subsystem $M_{T 2}$ for the $b \bar{b}$ system $\left(M_{T 2}^{(b)}\right)$ and the lepton system $\left(M_{T 2}^{(\ell)}\right)$ [13]. The variable $\hat{s}_{\min }^{(\mathrm{v})}$ is defined as $\hat{s}_{\text {min }}^{(\mathrm{v})}=m_{\mathrm{v}}^{2}+2\left(\sqrt{\left|\vec{P}_{T}^{\mathrm{v}}\right|^{2}+m_{\mathrm{v}}^{2}}\left|\overrightarrow{\boldsymbol{P}}_{T}\right|-\vec{P}_{T}^{\mathrm{v}} \cdot \overrightarrow{\boldsymbol{P}}_{T}\right)$ [14-16], where (v) represents a set of visible particles under consideration, while $m_{\mathrm{v}}$ and $\vec{P}_{T}^{\mathrm{v}}$ are their invariant mass and transverse momentum, respectively. It provides the minimum value of the Mandelstam invariant mass $\hat{s}$, which is consistent with the observed visible four-momentum vector. The $M_{T 2}$ is defined as $M_{T 2}(\tilde{m}) \equiv \min \left\{\max \left[M_{T P_{1}}\left(\vec{p}_{\nu T}, \tilde{m}\right), M_{T P_{2}}\left(\vec{p}_{\bar{\nu} T}\right.\right.\right.$, $\tilde{m})]\}$ where $\tilde{m}$ is the test mass for the daughter particle, and the minimization over the transverse masses of the parent particles $M_{T P_{i}}(i=1,2)$ is performed over the transverse neutrino momenta $\vec{p}_{\nu T}$ and $\vec{p}_{\bar{\nu} T}$ subject to the $\vec{p}_{T}$ constraint $[13,16,19]$.

Analysis.-Both the signal and the SM backgrounds were generated by MADGRAPH5_aMC@NLO [25] with the default NNPDF2.3QED parton distribution functions [26] in leading order QCD accuracy at the $\sqrt{s}=14 \mathrm{TeV}$ LHC. The default dynamical renormalization and factorization scales were used and off-shell effects for the top quark and $W$ boson are properly included. The double Higgs production cross section $\left(\sigma_{h h}=40.7 \mathrm{fb}\right)$ was normalized to the next-tonext-to-leading order (NNLO) accuracy in QCD [27]. Including all relevant branching fractions, we obtain $\sigma_{h h} \times$ $2 \times \mathrm{BR}(h \rightarrow b \bar{b}) \times \mathrm{BR}\left(h \rightarrow W W^{*} \rightarrow \ell^{+} \ell^{-} \nu \bar{\nu}\right)=0.648 \mathrm{fb}$, where $\ell$ denotes an electron or a muon, including leptons from tau decays. The major $t \bar{t}$ background is normalized to the NNLO QCD cross section $953.6 \mathrm{pb}$ [28]. The next dominant $t \bar{t} h$ background is normalized to the next-toleading order (NLO) QCD cross section $611.3 \mathrm{fb}$ [29], while for the $t \bar{t} V\left(V=W^{ \pm}, Z\right)$ background, we apply an NLO $k$ factor of 1.54 , resulting in $1.71 \mathrm{pb}$ [30]. A conservative NLO $k$ factor 2 is applied for Drell-Yan

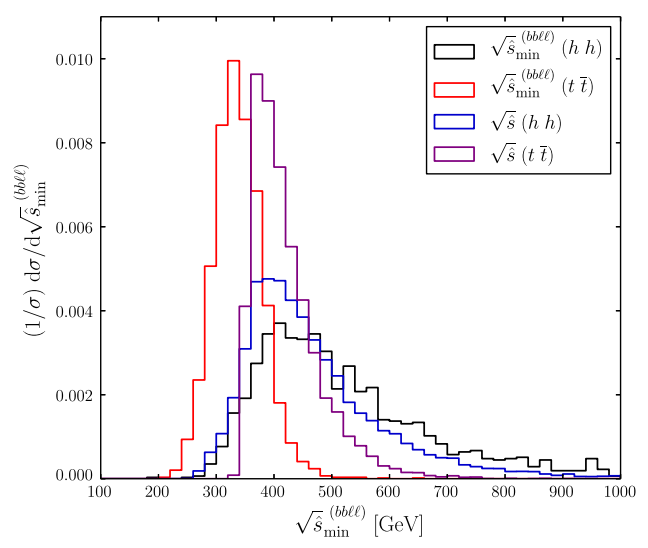

FIG. 1. Distributions of $\sqrt{\hat{s}}_{\text {min }}^{(\text {bbel })}$ and the true $\sqrt{\hat{s}}$ for the case of $h h$ and $t \bar{t}$ production. 

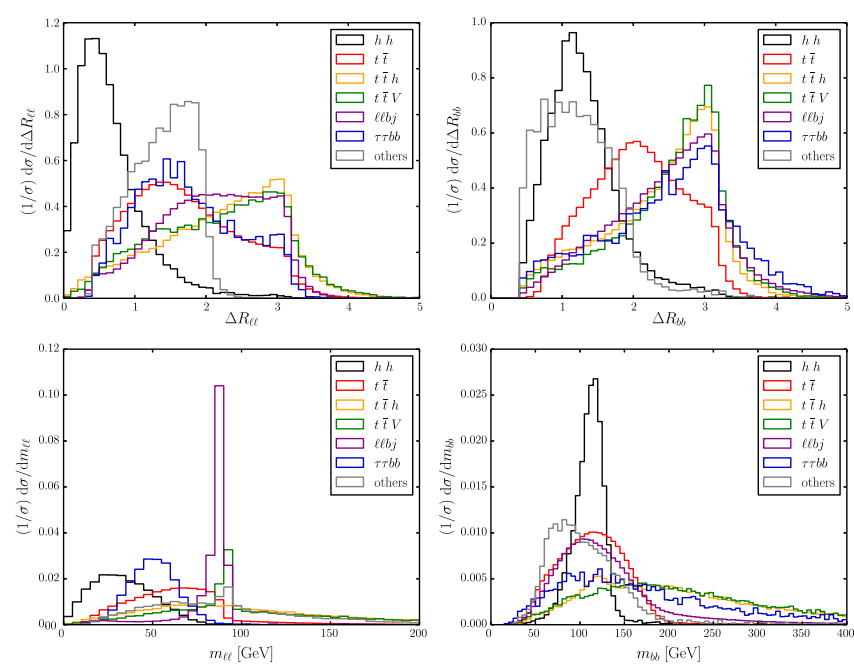

FIG. 2. Distributions of $\Delta R_{\ell \ell}, \Delta R_{b b}, m_{\ell \ell}$, and $m_{b b}$ after basic generation level cuts.

backgrounds $\ell \ell b j$ and $\tau \tau b b$, with $j$ representing partons in the five-flavor scheme. Note that a recent study shows $\mathrm{k}_{\mathrm{QCD} \otimes \mathrm{QED}}^{\mathrm{N} N \mathrm{DY}} \approx 1$ [31]. Finally we include the irreducible $j j \ell \ell \nu \bar{\nu}$ background from the mixed $\mathrm{QCD}+\mathrm{EW}$ process with $\mathrm{k}_{\mathrm{NLO}}=2$ (denoted as "others" in the rest of this Letter).

Both signal and background events are showered and hadronized by PYTHIA 6 [32]. Jets are clustered with the FASTJET [33] implementation of the anti- $k_{T}$ algorithm [34] with a fixed cone size of $R=0.4$ for a jet, where the $R$ is the distance from the origin in the $(\eta, \phi)$ space in terms of the pseudorapidity $\eta$ and the azimuthal angle $\phi$. We include semirealistic detector effects relevant for the HL-LHC based on Ref. [35], where jets, leptons, and $\boldsymbol{E}_{T}$ are smeared according to their energies.

We require at least two jets with $p_{T}>30 \mathrm{GeV}$ and $\left|\eta^{j}\right|<2.5$, and exactly two leptons with $p_{T}^{\ell}>20 \mathrm{GeV}$, $\left|\eta^{\ell}\right|<2.5$. For the lepton isolation criteria, we impose $p_{T}^{\ell} / p_{T}^{\Sigma}>0.7$ where $p_{T}^{\Sigma}$ is the scalar sum of transverse momenta of final state particles (including the lepton itself) within $\Delta R=0.3$. We further require that the two leading jets should be $b$ tagged. For $b$-tagging efficiency, we take a flat efficiency of $\epsilon_{b \rightarrow b}=0.7$ and a mistag rate from a $c$ jet (light jet) as $\epsilon_{c \rightarrow b}=0.2\left(\epsilon_{j \rightarrow b}=0.01\right)$ [36].
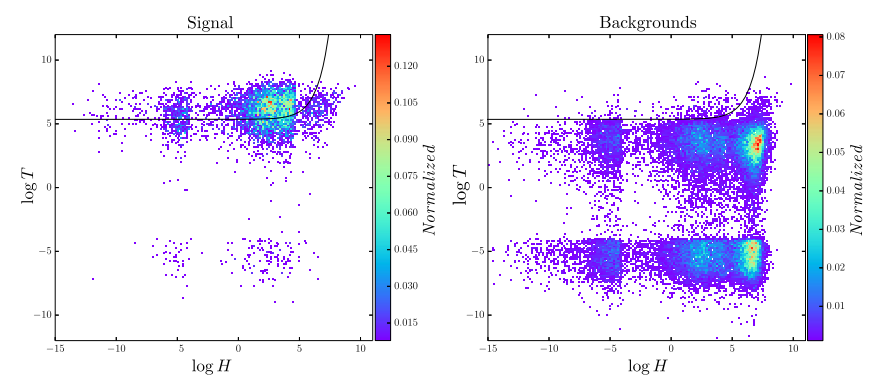

FIG. 3. Scatter distribution of $(\log H, \log T)$ for signal $(h h)$ and backgrounds ( $t \bar{t}, t \bar{t} h, t \bar{t} V, \ell \ell b j, \tau \tau b b$, and others) after loose baseline selection cuts. The curves are the optimized cuts as in Table I.

Figure 1 shows distributions of $\sqrt{\hat{s}}_{\min }^{(b b \ell)}$ and the true $\sqrt{\hat{s}}$ for $h h$ and $t \bar{t}$ events. First, we observe that $\sqrt{\hat{s}}_{\min }^{(b b \ell)}(h h)$ provides a good measure of the true $\sqrt{\hat{s}}(h h)$, while $\sqrt{\hat{s}}_{\text {min }}^{(b b \ell \ell)}(t \bar{t})$ peaks lower, near the $2 m_{t}$ threshold. Second, both $\sqrt{\hat{s}}(h h)$ and $\sqrt{\hat{s}}(t \bar{t})$ peak at $\sim 400 \mathrm{GeV}$. This implies that, while the two top quarks are produced near threshold $\left(2 m_{t}\right)$, the two Higgs bosons are produced well above the corresponding $2 m_{h}$ threshold. Consequently, the two top quarks are more or less at rest, while the two Higgs bosons are boosted and their decay products tend to be more collimated. This observation motivates the use of the variables $\Delta R_{\ell \ell}, \Delta R_{b b}, m_{\ell \ell}$, and $m_{b b}$ for our starting cuts (their individual distributions are shown in Fig. 2). These cuts, along with cuts on $\vec{p}_{T}$ and the lepton transverse momenta $p_{T}^{\ell}$, provide our baseline cuts. Table I lists the corresponding signal and background cross sections (first row). We then compute Topness and Higgsness for each event, which provides a pair of likelihoods in the $(\log H$, $\log T$ ) space. Our results are shown in Fig. 3, where the Higgsness and Topness are chosen as the $x$ axis and $y$ axis, respectively. The $t \bar{t}$ events are expected to be in the bottom right corner (see the right panel), while the $h h$ events are expected to have smaller Higgsness and higher Topness (see the left panel). This motivates the use of a curve in the ( $\log H$, $\log T$ ) space as a cut in order to separate signal and background.

The second row in Table I lists the signal and background cross sections after some additional cuts. The last

TABLE I. Signal and background cross sections in fb after baseline cuts (first row) and additional cuts (second row), considering Higgsness $\oplus$ Topness $\oplus M_{T 2}^{(b)} \oplus M_{T 2}^{(\ell)} \oplus \sqrt{\hat{s}}_{\text {min }}^{(\ell \ell)}$ requiring $\mathrm{N}_{\text {sig }}^{\mathrm{SM}}=20$. The significance $\sigma$ is calculated using the log-likelihood ratio for a luminosity of $3 \mathrm{ab}^{-1}$.

\begin{tabular}{lcccccccccc}
\hline \hline & Signal & $t \bar{t}$ & $t \bar{t} h$ & $t \bar{t} V$ & $\ell \ell b j$ & $\tau \tau b b$ & others & $\sigma$ & $\mathrm{N}_{\mathrm{sig}}^{\mathrm{SM}} / \mathrm{N}_{\mathrm{bknd}}$ \\
\hline Baseline selections: $\mathbb{E}_{T}>20 \mathrm{GeV}, p_{T}^{\ell}>20 \mathrm{GeV}$, & 0.0124 & 1.1724 & 0.0297 & 0.0246 & 0.0158 & 0.0379 & 0.00590 & 0.60 & 0.00964 \\
& $\Delta R_{\ell \ell}<1.0, m_{\ell \ell}<65 \mathrm{GeV}, \Delta R_{b b}<1.3$, \\
& & & & & & & & & \\
& & & & & & & & & & \\
Higgsness $\oplus$ Topness $\oplus M_{T 2}^{(b)} \oplus 140 \mathrm{GeV}$ & $M_{T 2}^{(\ell)} \oplus \sqrt{\hat{s}}_{\min }^{(\ell \ell)}$ & 0.0065 & 0.0090 & 0.0073 & 0.0051 & $2.4 \times 10^{-4}$ & 0.0046 & 0.00185 & 2.06 & 0.23 \\
\hline \hline
\end{tabular}



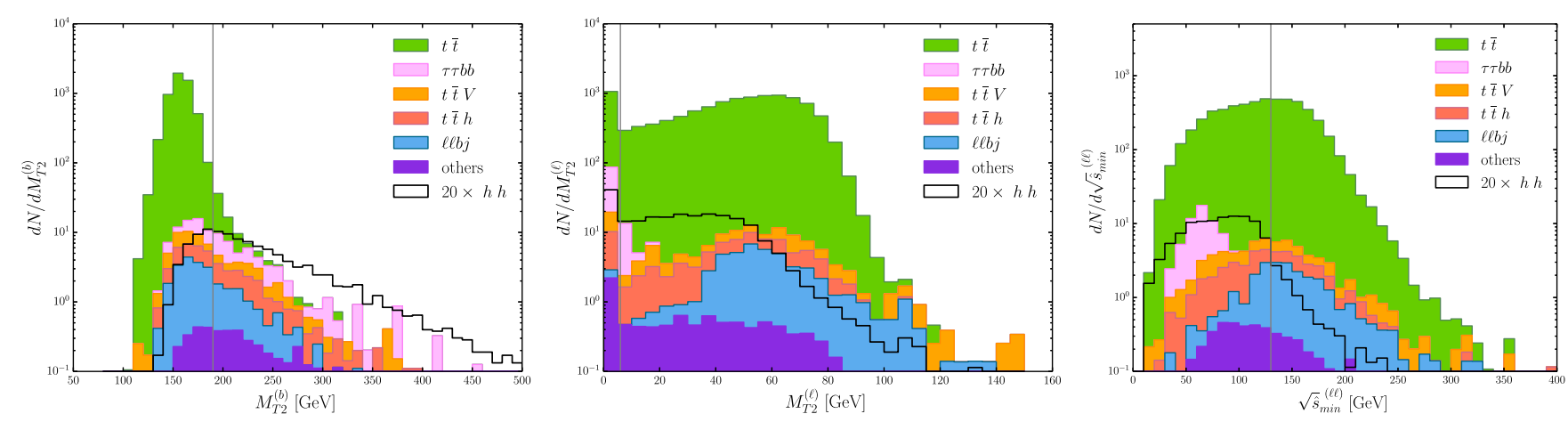

FIG. 4. Distributions for signal $(h h)$ and all backgrounds $\left(t \bar{t}, t \bar{t} h, t \bar{t} V, \ell \ell b j, \tau \tau b b\right.$, and others) for $M_{T 2}^{(b)}, M_{T 2}^{(\ell)}$, and $\sqrt{\hat{s}}_{\min }^{(\ell \ell)}$ after loose baseline selection cuts. The vertical lines at $M_{T 2}^{(b)}=190 \mathrm{GeV}, M_{T 2}^{(\ell)}=6 \mathrm{GeV}$, and $\sqrt{\hat{s}}_{\text {min }}^{(\ell \ell)}=130 \mathrm{GeV}$ are the optimized cuts from Table I.

two columns show the corresponding signal significance using the log-likelihood ratio method for a luminosity of $3 \mathrm{ab}^{-1}$ and the signal-over-background ratio $\mathrm{N}_{\text {sig }}^{\mathrm{SM}} / \mathrm{N}_{\text {bknd }}$, respectively. Our baseline cuts result in a significance of 0.6 with $\mathrm{N}_{\text {sig }}^{\mathrm{SM}}=37$ and $\mathrm{N}_{\mathrm{bknd}}=3841$, which is in rough agreement with results in literature $[9,12]$. We note that one can enhance the signal sensitivity by using Higgsness $\oplus$ Topness along with $M_{T 2}^{(b)}, \quad M_{T 2}^{(\ell)}$, and $\sqrt{\hat{s}}_{\min }^{(\ell \ell)}$. The $M_{T 2}^{(b)}\left(M_{T 2}^{(\ell)}\right)$ is $M_{T 2}$ computed for a subsystem where the two $b$ quarks (leptons) are considered as the visible particles and the two $W \mathrm{~s}(\nu \mathrm{s})$ as the invisible particles with $\tilde{m}=m_{W}(\tilde{m}=0)$. The $M_{T 2}^{(b)}$ for $t \bar{t}$ events has a kinematic endpoint at $m_{t}$, while the distributions for the other processes may extend beyond this endpoint, as shown in the left panel of Fig. 4. Similarly, $M_{T 2}^{(\ell)}$ has an endpoint at $m_{W}$ for $t \bar{t}$ and at $m_{\tau}$ for $\tau \tau b b$, as shown in the middle panel of Fig. 4. Finally, $\sqrt{\hat{s}}_{\text {min }}^{(\ell \ell)}$ in the right panel shows an endpoint at $m_{h}$ for $h h$ production, while all other backgrounds extend above this point. The cuts on Higgsness $\oplus$ Topness, together with the cuts on $M_{T 2}^{(b)}$, $M_{T 2}^{(\ell)}$, and $\sqrt{\hat{s}}_{\text {min }}^{(\ell \ell)}$ increase the significance up to $\sigma=2.1$ and $\mathrm{N}_{\text {sig }}^{\mathrm{SM}} / \mathrm{N}_{\text {bknd }}=0.25$, keeping $\mathrm{N}_{\text {sig }}^{\mathrm{SM}}=20$. The curve in the two scatter plots of Fig. 3 and the vertical lines at $M_{T 2}^{(b)}=190 \mathrm{GeV}, M_{T 2}^{(\ell)}=6 \mathrm{GeV}$, and $\sqrt{\hat{s}}_{\min }^{(\ell \ell)}=130 \mathrm{GeV}$ in Fig. 4 represent the optimized cuts from the second row of Table I.

A higher significance of $3.0 \sigma$ can be obtained by imposing slightly tighter baseline cuts $\left(\Delta R_{\ell \ell}<0.48\right.$, $m_{\ell \ell}<60 \mathrm{GeV}, \Delta R_{b b}<1.1$, and $95<m_{b b}<140 \mathrm{GeV}$ ) and reoptimizing the additional cuts for $\mathrm{N}_{\text {sig }}^{\mathrm{SM}}=10$ and $\mathrm{N}_{\text {sig }}^{\mathrm{SM}} / \mathrm{N}_{\text {bknd }} \sim 1$ 1.2. Conversely, we can obtain more signal events with slightly looser baseline cuts. For instance, $\mathrm{N}_{\text {sig }}^{\mathrm{SM}}=35$ with $\mathrm{N}_{\text {sig }}^{\mathrm{SM}} / \mathrm{N}_{\mathrm{bknd}}=0.061$ is easily obtained with loose baseline cuts $\left(\Delta R_{\ell \ell}<1.6, \quad m_{\ell \ell}<75 \mathrm{GeV}\right.$, $\Delta R_{b b}<1.5$, and $\left.90<m_{b b}<140 \mathrm{GeV}\right)$ and optimization of Higgsness $\oplus$ Topness, which gives a significance of
$1.4 \sigma$. This can be compared to existing results with a similar number of signal events but a lower significance of $\sim 0.7[9,12]$.

Finally, we perform a similar analysis for different values of $\kappa_{3}$, and show the expected signal significance as a function of $\kappa_{3}$ in Fig. 5. We obtain each curve by applying the same set of cuts optimized for the SM point $\left(\kappa_{3}=1\right)$ to non-SM points $\left(\kappa_{3} \neq 1\right)$ for $\mathrm{N}_{\text {sig }}^{\mathrm{SM}}=35$ in black, $\mathrm{N}_{\text {sig }}^{\mathrm{SM}}=20$ in red, and $\mathrm{N}_{\text {sig }}^{\mathrm{SM}}=10$ in blue. The red curve (middle) is the result of Table I for $\mathrm{N}_{\text {sig }}^{\mathrm{SM}}=20$, while the other two curves correspond to tighter (blue) and looser (black) baseline selections for $\mathrm{N}_{\text {sig }}^{\mathrm{SM}}=35$ and $\mathrm{N}_{\text {sig }}^{\mathrm{SM}}=10$, respectively. One can easily compute the number of signal events for $\kappa_{3} \neq 1$,

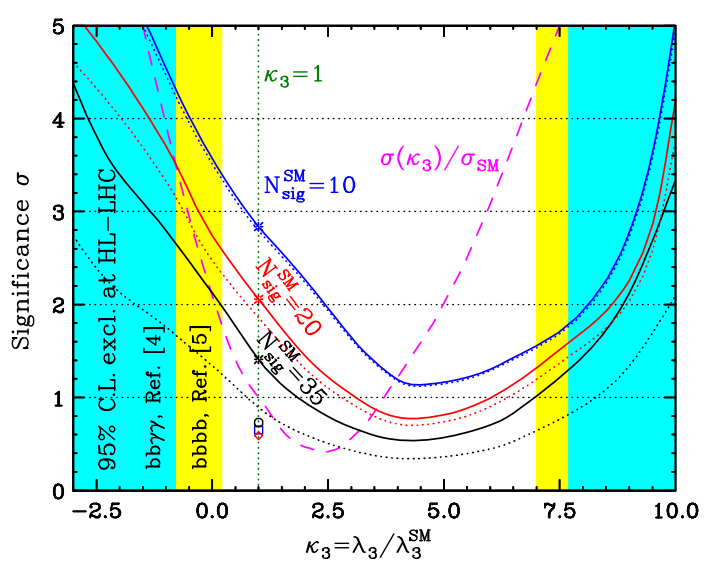

FIG. 5. Expected significance $\sigma$ at the $14 \mathrm{TeV}$ LHC with $3 \mathrm{ab}^{-1}$ as a function of the triple Higgs coupling $\kappa_{3}$. We obtain each curve by applying the same set of cuts optimized for the SM point $\left(\kappa_{3}=1\right)$ to non-SM points $\left(\kappa_{3} \neq 1\right)$ for $\mathrm{N}_{\text {sig }}^{\mathrm{SM}}=35$ in black, $\mathrm{N}_{\text {sig }}^{\mathrm{SM}}=20$ in red, and $\mathrm{N}_{\text {sig }}^{\mathrm{SM}}=10$ in blue. The yellow (cyan) shade represents 95\% C.L. exclusion of $\kappa_{3}$ in the $b b \gamma \gamma$ channel [4] ( $b b b b$ channel [5]) at the HL-LHC. The three marks $\diamond, \bigcirc$, and $\square$ display the signal significance using CMS-NN [9], CMS-BDT [11], and BDT [12], respectively. The dotted curves represent the significance including $5 \%$ systematic uncertainty. 
using the ratio of cross sections of $\sigma\left(\kappa_{3} \neq 1\right)$ to $\sigma_{\mathrm{SM}}=$ $\sigma\left(\kappa_{3}=1\right)$ (dashed, magenta) [8] and $\mathrm{N}_{\mathrm{sig}}^{\mathrm{SM}}$ for each $\mathrm{SM}$ point.

At 95\% confidence level (C.L.), the Higgs boson selfcoupling is expected to be constrained to $-0.8<\kappa_{3}<7.7$ $\left(0.2<\kappa_{3}<7.0\right)$ from the $b b \gamma \gamma$ final state [4] (bbbb [5]) at the HL-LHC, which are shown as yellow and cyan shade, respectively (see Ref. [37] for an improved projection in the $b b \gamma \gamma$ channel). The three symbols $\diamond, \bigcirc$, and $\square$ represent the significance $\sigma=0.6$ using CMS-NN [9], $\sigma=0.73$ using CMS-BDT [11], and $\sigma=0.62$ using BDT [12], respectively. Our method clearly shows better performance for both signal sensitivity and exclusion.

Discussion.-We have investigated the discovery potential of nonresonant double Higgs production in the final state with $b \bar{b} \ell^{+} \ell^{-}+\mathbb{E}_{T}$. We have shown that the signal sensitivity can be significantly improved by using suitable kinematic variables, without relying on sophisticated methods. Our only assumptions are event topologies of signal and backgrounds with appropriate mass shell conditions. Yet, our final signal significance is higher than existing results in literature at least by a factor of 2 , due to correct use of kinematics for the corresponding event topologies. One could even make further improvement by optimizing cut values and parameters in the discussed method.

Among various sources of systematic uncertainties, a current CMS study [10] lists the QCD scale uncertainty (13\%) and the uncertainty in the $t \bar{t}$ cross section (5\%), as the dominant ones, and we estimate the final systematic uncertainty to be about $15 \%$ at the $13 \mathrm{TeV}$ LHC. It may be reduced to $\sim 5 \%$ at the HL-LHC [9], whose effects are shown in the dotted curves in Fig. 5. Our result including the systematic uncertainty still remains very optimistic, given that $\mathrm{N}_{\text {sig }}^{\mathrm{SM}} / \mathrm{N}_{\text {bknd }}$ is large. Finally, pileup effects may be mitigated by installing new precision timing detectors [38] or by analysis techniques using substructure [39-42] or machine learning $[43,44]$. In particular, the charged leptons are free of pileup contamination, which further motivates the study of our final state.

The current observed upper limit on the SM $h h \rightarrow$ $b \bar{b} V V \rightarrow b \bar{b} \ell^{+} \ell^{-} \nu \bar{\nu}$ cross section is found to be $72 \mathrm{fb}$ by CMS using DNN with $36 \mathrm{fb}^{-1}$ of luminosity [10]. We believe our method can be used in order to improve the signal sensitivity already at the $13 \mathrm{TeV}$ LHC.

We thank Minho Kim for a helpful discussion on BDT and the anonymous referee for constructive comments. K. K. thanks the Aspen Center for Physics for hospitality during the completion of this Letter, supported in part by National Science Foundation Grant No. PHY-1607611. This work is supported in part by United States Department of Energy (DE-SC0010296, DE-SC0017988, and DE-SC0017965) and Korea NRF2018R1C1B6006572.
[1] G. Aad et al. (ATLAS Collaboration), Phys. Lett. B 716, 1 (2012).

[2] S. Chatrchyan et al. (CMS Collaboration), Phys. Lett. B 716, 30 (2012).

[3] G. Aad et al. (ATLAS and CMS Collaborations), J. High Energy Phys. 08 (2016) 045.

[4] ATLAS Collaboration, Report No. ATL-PHYS-PUB-2017001.

[5] ATLAS Collaboration, Report No. ATL-PHYS-PUB-2016024.

[6] J. H. Kim, Y. Sakaki, and M. Son, Phys. Rev. D 98, 015016 (2018).

[7] CMS Collaboration, Report No. CMS-PAS-HIG-17-030.

[8] J. Baglio, A. Djouadi, R. Grber, M. M. Mhlleitner, J. Quevillon, and M. Spira, J. High Energy Phys. 04 (2013) 151.

[9] CMS Collaboration, Report No. CMS-PAS-FTR-15-002.

[10] A. M. Sirunyan et al. (CMS Collaboration), J. High Energy Phys. 01 (2018) 054.

[11] CMS Collaboration, Report No. CMS-PAS-FTR-16-002.

[12] A. Adhikary, S. Banerjee, R. K. Barman, B. Bhattacherjee, and S. Niyogi, J. High Energy Phys. 07 (2018) 116.

[13] M. Burns, K. Kong, K. T. Matchev, and M. Park, J. High Energy Phys. 03 (2009) 143.

[14] P. Konar, K. Kong, K. T. Matchev, and M. Park, J. High Energy Phys. 06 (2011) 041.

[15] P. Konar, K. Kong, and K. T. Matchev, J. High Energy Phys. 03 (2009) 085.

[16] A. J. Barr, T. J. Khoo, P. Konar, K. Kong, C. G. Lester, K. T. Matchev, and M. Park, Phys. Rev. D 84, 095031 (2011).

[17] A. J. Barr, M. J. Dolan, C. Englert, and M. Spannowsky, Phys. Lett. B 728, 308 (2014).

[18] M. L. Graesser and J. Shelton, Phys. Rev. Lett. 111, 121802 (2013).

[19] C. G. Lester and D. J. Summers, Phys. Lett. B 463, 99 (1999).

[20] F. James and M. Roos, Comput. Phys. Commun. 10, 343 (1975).

[21] T. Han, I. W. Kim, and J. Song, Phys. Lett. B 693, 575 (2010).

[22] T. Han, I. W. Kim, and J. Song, Phys. Rev. D 87, 035004 (2013).

[23] T. Han, I. W. Kim, and J. Song, Phys. Rev. D 87, 035003 (2013).

[24] W. S. Cho, D. Kim, K. T. Matchev, and M. Park, Phys. Rev. Lett. 112, 211801 (2014).

[25] J. Alwall, R. Frederix, S. Frixione, V. Hirschi, F. Maltoni, O. Mattelaer, H.-S. Shao, T. Stelzer, P. Torrielli, and M. Zaro, J. High Energy Phys. 07 (2014) 079.

[26] R. D. Ball, V. Bertone, S. Carrazza, L. Del Debbio, S. Forte, A. Guffanti, N. P. Hartland, and J. Rojo (NNPDF Collaboration), Nucl. Phys. B877, 290 (2013).

[27] J. Grigo, K. Melnikov, and M. Steinhauser, Nucl. Phys. B888, 17 (2014).

[28] M. Czakon, P. Fiedler, and A. Mitov, Phys. Rev. Lett. 110, 252004 (2013).

[29] S. Dittmaier et al. (LHC Higgs Cross Section Working Group), arXiv:1101.0593.

[30] D. de Florian et al. (LHC Higgs Cross Section Working Group), arXiv:1610.07922.

[31] D. de Florian, M. Der, and I. Fabre, Phys. Rev. D 98, 094008 (2018). 
[32] T. Sjostrand, S. Mrenna, and P. Z. Skands, J. High Energy Phys. 05 (2006) 026.

[33] M. Cacciari, G. P. Salam, and G. Soyez, Eur. Phys. J. C 72, 1896 (2012).

[34] M. Cacciari, G. P. Salam, and G. Soyez, J. High Energy Phys. 04 (2008) 063.

[35] ATLAS Collaboration, Report No. ATL-PHYS-PUB-2013004.

[36] A. Sirunyan et al. (CMS Collaboration), J. Instrum. 13, P05011 (2018).

[37] F. Kling, T. Plehn, and P. Schichtel, Phys. Rev. D 95, 035026 (2017).
[38] CMS Collaboration, Report No. CERN-LHCC-2017-027, https://cds.cern.ch/record/2296612.

[39] L. Asquith et al., arXiv:1803.06991.

[40] G. Soyez, arXiv:1801.09721.

[41] G. Aad et al. (ATLAS Collaboration), J. High Energy Phys. 05 (2012) 128.

[42] D. Bertolini, P. Harris, M. Low, and N. Tran, J. High Energy Phys. 10 (2014) 059.

[43] P. T. Komiske, E. M. Metodiev, B. Nachman, and M. D. Schwartz, J. High Energy Phys. 12 (2017) 051.

[44] J. Arjona Martnez, O. Cerri, M. Pierini, M. Spiropulu, and J. R. Vlimant, arXiv:1810.07988. 